\title{
Magnetohydrodynamics as Superfluidity
}

\author{
Jay Armas ${ }^{1,2, *}$ and Akash Jain ${ }^{3, \dagger}$ \\ ${ }^{1}$ Institute for Theoretical Physics, University of Amsterdam, 1090 GL Amsterdam, Netherlands \\ ${ }^{2}$ Dutch Institute for Emergent Phenomena, 1090 GL Amsterdam, Netherlands \\ ${ }^{3}$ Centre for Particle Theory \& Department of Mathematical Sciences, Durham University, Durham DH1 3LE, United Kingdom
}

(Received 31 August 2018; revised manuscript received 10 December 2018; published 9 April 2019)

\begin{abstract}
We show that relativistic magnetohydrodynamics (MHD) can be recast as a novel theory of superfluidity. This new theory formulates MHD just in terms of conservation equations, including dissipative effects, by introducing appropriate variables such as a magnetic scalar potential, and providing necessary and sufficient conditions to obtain equilibrium configurations. We show that this scalar potential can be interpreted as a Goldstone mode originating from the spontaneous breaking of a one-form symmetry, and present the most generic constitutive relations at one derivative order for a parity-preserving plasma in this new superfluid formulation.
\end{abstract}

DOI: 10.1103/PhysRevLett.122.141603

Relativistic magnetohydrodynamics (MHD) provides a universal framework to study plasma physics in astrophysical settings as well as in laboratory experiments [1]. As an effective theory for interactions between electromagnetic and thermal degrees of freedom of matter, MHD describes the coupling of Maxwell's equations to hydrodynamics. For most of its applications, MHD is formulated under the assumption that the electric fields are Debye screened and are therefore weak or short ranged, while the magnetic fields could be arbitrary, and that the plasma is electrically neutral at hydrodynamic length scales. Formulations of MHD under these assumptions have been extensively studied and have a wide range of applicability. However, the structural foundations and transport properties of MHD have only recently received considerable attention [2-5]. The main purpose of this Letter is to present an equivalent formulation of dissipative MHD as a system of (higherform) conservation equations, which is better suited for numerical analyses, and to resolve certain underlying technical issues in the definition of hydrostatic equilibrium.

Traditional treatments of MHD are formulated in terms of a stress tensor $T^{\mu \nu}$ and charge current $J^{\mu}$, subject to energy-momentum conservation, Maxwell's equations, and Bianchi identity (e.g., see Ref. [4]),

$$
\nabla_{\mu} T^{\mu \nu}=F^{\nu \rho} J_{\rho}, \quad J^{\mu}+J_{\mathrm{ext}}^{\mu}=0, \quad \epsilon^{\mu \nu \rho \sigma} \nabla_{\nu} F_{\rho \sigma}=0 .
$$

Published by the American Physical Society under the terms of the Creative Commons Attribution 4.0 International license. Further distribution of this work must maintain attribution to the author(s) and the published article's title, journal citation, and DOI. Funded by SCOAP.
Here, the components of $F_{\mu \nu}$ are the electromagnetic fields and $J_{\text {ext }}^{\mu}$ is a conserved external charge source (e.g., a lattice of ions) satisfying $\nabla_{\mu} J_{\text {ext }}^{\mu}=0$. The charge current $J^{\mu}$ can be split into $\nabla_{\nu} F^{\nu \mu}+J_{\text {matter }}^{\mu}$, where $J_{\text {matter }}^{\mu}$ is the contribution from matter fields, converting Maxwell's equations into their better known form. The Bianchi identity is solved by introducing the photon field such that $F_{\mu \nu}=2 \partial_{[\mu} A_{\nu]}$, while the remaining 8 equations govern the dynamics of the temperature $T$, chemical potential $\mu$, fluid velocity $u^{\mu}$, and gauge field $A_{\mu}$. In usual MHD applications, $J_{\mathrm{ext}}^{\mu}$ is taken to be zero while $\mu$ is fixed such that the electric charge vanishes at hydrodynamic length scales.

In practice, however, for instance in numerical approaches, Maxwell's equations can be used to eliminate $\mu$ and Debyescreened electric fields $E_{\mu}=F_{\mu \nu} u^{\nu}$ from the system, leaving $u^{\mu}, T$, and magnetic fields $B^{\mu}=\frac{1}{2} \epsilon^{\mu \nu \rho \sigma} u_{\nu} F_{\rho \sigma}$ to be the only relevant fields. The dynamics for $u^{\mu}$ and $T$ is governed by energy-momentum conservation, while that for $B^{\mu}$ by the Bianchi identity (see, e.g., Refs. [6,7]).

Extrapolating this line of thought, the authors of Ref. [3] (see also Ref. [2]), inspired by the framework of generalized global symmetries [8], proposed a formulation of MHD in terms of string or one-form fluids [2-5,8,9]. The key observation is that once Maxwell's equations in Eq. (1) are explicitly solved by setting $J^{\mu}=-J_{\text {ext }}^{\mu}$, the dynamics of MHD effectively reduces to that of a fluid with a global one-form symmetry. To wit, by defining a two-form current $J^{\mu \nu}=\frac{1}{2} \epsilon^{\mu \nu \rho \sigma} F_{\rho \sigma}$, and identifying the external charge source as $J_{\text {ext }}^{\mu}=\frac{1}{6} \epsilon^{\mu \nu \rho \sigma} H_{\nu \rho \sigma}$, where $H_{\mu \nu \rho}=3 \partial_{[\mu} b_{\nu \rho]}$ is the field strength associated with a background two-form gauge field $b_{\mu \nu}$, Eq. (1) can be rewritten as

$$
\nabla_{\mu} T^{\mu \nu}=\frac{1}{2} H^{\nu \rho \sigma} J_{\rho \sigma}, \quad \nabla_{\mu} J^{\mu \nu}=0 .
$$


The dynamical variables of MHD in this string fluid formulation are the string chemical potential $\varpi$ and the vector characterizing the direction of strings $h^{\mu}$ (with $h^{\mu} h_{\mu}=1$ and $u^{\mu} h_{\mu}=0$ ), in addition to $u^{\mu}$ and $T$. Heuristically, $h^{\mu}$ corresponds to the direction of magnetic fields, while $\varpi$ is a chemical potential conjugate to their strength

$$
\varpi=-2|B| \frac{\partial P}{\partial B^{2}}+\mathcal{O}(\partial), \quad h^{\mu}=\frac{B^{\mu}}{|B|}+\mathcal{O}(\partial),
$$

where $P\left(T, B^{2}\right)$ is the pressure of MHD. The string fluid pressure defined later in Eq. (5) is related to MHD pressure as $p(T, \varpi)=P\left(T, B^{2}\right)-2|B|^{2} \partial P / \partial B^{2}+\mathcal{O}(\partial)$. These relations admit corrections at higher derivative orders.

If we switch off the sources $H_{\mu \nu \lambda}=0$, Eq. (2) with the ideal order constitutive relations (5) and equation of state $P\left(T, B^{2}\right)=P(T)-\frac{1}{2} B^{2}$ or $p(T, \varpi)=P(T)+\frac{1}{2} \varpi^{2}$ reduces to the system of equations given in Ref. [6]. The first equation is the well-known energy-momentum conservation. The spatial components of the second equation can be seen as the induction equation, while the time component as the no-monopole constraint of Ref. [6].

As pointed out in Ref. [5], the string fluid variables $\varpi$ and $h^{\mu}$, while consistent, are not well suited for describing equilibrium configurations in MHD. In particular, a generic string fluid equilibrium configuration cannot be derived from a hydrostatic partition function within the framework of Ref. [3]. Such equilibrium configurations serve as initial conditions in numerical simulations of hydrodynamics, so it is crucial that we identify the appropriately suited degrees of freedom. In this Letter, we introduce a more natural pair of fields: gauge-non-invariant one-form chemical potential $\mu_{\mu}$ and "scalar Goldstone" $\varphi$ such that

$$
\varpi h_{\mu}=\mu_{\mu}-T \partial_{\mu} \varphi .
$$

When coupled to a time-independent background, we find that in equilibrium $\mu_{\mu} / T=b_{t \mu}$, while $\varphi$ plays a role similar to that of a "magnetic scalar potential" and is solved for using the no-monopole constraint. Drawing a comparison with the Goldstone phase field in typical superfluids, we formulate a novel theory of one-form superfluidity, where the underlying global one-form symmetry is spontaneously broken leading to a one-form Goldstone mode $\varphi_{\mu}[8,10,11]$. We show that the existence of this mode gives rise to a welldefined hydrostatic sector for string fluids, when viewed as a limit of one-form superfluids where only a part of the one-form symmetry is broken, with the associated scalar Goldstone $\varphi=u^{\mu} \varphi_{\mu} / T$.

Finally, while the traditional and string fluid formulations of MHD can easily be shown to be equivalent at ideal order as described above, at higher derivative orders this equivalence is quite nontrivial. It has only been established in the dissipative sector for linear fluctuations (Kubo formulas) in a state with $\mu=0$ [4]. We show an exact correspondence between MHD and our improved formulation of string fluids. The crucial ingredients of this correspondence are presented in this Letter, while further details are relegated to a companion publication [12].

String fluids and equilibrium.-One-form hydrodynamics is governed by the equations of motion (2) and respective constitutive relations, that is, the most generic expressions for $\left(T^{\mu \nu}, J^{\mu \nu}\right)$ in terms of $\left(T, u^{\mu}, \varpi, h^{\mu}\right)$ and $\left(g_{\mu \nu}, b_{\mu \nu}\right)$ allowed by symmetries and the second law of thermodynamics. At ideal order, these relations read

$$
\begin{aligned}
T^{\mu \nu} & =(\epsilon+p) u^{\mu} u^{\nu}+p g^{\mu \nu}-\varpi \rho h^{\mu} h^{\nu}+\mathcal{O}(\partial), \\
J^{\mu \nu} & =2 \rho u^{[\mu} h^{\nu]}+\mathcal{O}(\partial),
\end{aligned}
$$

where $p(T, \varpi)$ is an arbitrary function, while $\epsilon(T, \varpi)$ and $\rho(T, \varpi)$ are determined by the thermodynamic relations $\epsilon+p=T s+\varpi \rho$ and $d p=s d T+\rho d \varpi$. The associated entropy current $S^{\mu}=s u^{\mu}$ is trivially conserved (see Ref. [3] for more details).

Hydrodynamics is the study of small fluctuations of a quantum system around thermodynamic equilibrium and hence it is important to understand how to describe equilibrium configurations. As usual, one assumes the existence of an arbitrary time coordinate $t$ such that $g_{t t}<0, \partial_{t} g_{\mu \nu}=\partial_{t} b_{\mu \nu}=0$, and $u^{\mu} / T=\delta_{t}^{\mu}$. However, this is not sufficient to attain equilibrium in string fluids since the conservation of string charge $\nabla_{\mu}\left(T \rho h^{\mu}\right)=0$, arising from the no-monopole constraint in Eq. (2), is not satisfied. To circumvent this issue, the authors of Ref. [3] specialized to backgrounds that further admit a spatial coordinate $z$ such that $\partial_{z} g_{\mu \nu}=\partial_{z} b_{\mu \nu}=0$ with $g_{t z}=0$ and obtained an equilibrium solution by setting $h^{\mu}=\delta_{z}^{\mu} / \sqrt{g_{z z}}$ and $\varpi / T=b_{t z} / \sqrt{g_{z z}}$. However, this "equilibrium solution," besides being only a subset of the solutions to $\nabla_{\mu}\left(T \rho h^{\mu}\right)=0$, generically contributes to entropy production [5], which an equilibrium fluid configuration, by definition, cannot. For example, consider a particular dissipative correction to $J^{\mu \nu}$ obtained in Ref. [3],

$$
\delta J_{(1)}^{\mu \nu} \ni-r_{\|} \Delta^{\mu \rho} \Delta^{\nu \sigma}\left[2 T \nabla_{[\rho}\left(\frac{\varpi}{T} h_{\sigma]}\right)+u_{\lambda} H_{\rho \sigma}^{\lambda}\right] .
$$

Here, $r_{\|} \geq 0$ is a dissipative transport coefficient and $\Delta^{\mu \nu}=g^{\mu \nu}+u^{\mu} u^{\nu}-h^{\mu} h^{\nu}$. It may be explicitly checked that this term does not vanish when evaluated on the equilibrium solution of Ref. [3]. Therefore, it must be imposed to vanish by hand as an ad hoc constraint on equilibrium backgrounds, in addition to requiring an isometry along the coordinate $z$. An infinite cascade of similar conditions show up at every derivative order [5]. Thus, in contrast to the case of typical charged fluids, the hydrostatic sector of string fluids is ill defined. We show that there is a first-principles derivation of equilibrium 
configurations that do not require ad hoc constraints nor existence of a preferred coordinate $z$.

Revisiting string fluids.- Some of the issues mentioned have an analogue in superfluid dynamics. In this context, had we considered the components of the superfluid velocity $\xi^{\mu}$ as fundamental degrees of freedom, ignorant of its definition $\xi_{\mu}=\partial_{\mu} \phi+A_{\mu}$ in terms of the Goldstone mode $\phi$, we could be tempted to introduce a preferred $z$ coordinate in equilibrium to align $\xi^{\mu}$ with. However, it is precisely $\phi$ that leads to well-defined equilibrium configurations for superfluids [13].

These considerations lead us to reevaluate whether the string fluid variables $\left(T, u^{\mu}, \varpi, h^{\mu}\right)$ describe a symmetryunbroken phase or if the underlying one-form symmetry is spontaneously broken. In order to identify the correct hydrodynamic fields in a symmetry-unbroken phase, we follow the approach of Ref. [14] for usual charged fluids. In this setting, the fields $\left(T, u^{\mu}, \mu\right)$ can be exchanged by a set of symmetry parameters $\mathcal{B}=\left(\beta^{\mu}, \Lambda^{\beta}\right)$, where $\beta^{\mu}=u^{\mu} / T$ and $\Lambda^{\beta}=\mu / T-\beta^{\mu} A_{\mu}$. Under the action of an infinitesimal symmetry transformation $\mathcal{X}=\left(\chi^{\mu}, \Lambda^{\chi}\right)$, with $\chi^{\mu}$ being a diffeomorphism and $\Lambda^{\chi}$ a gauge transformation, they transform according to $\delta_{\mathcal{X}} \beta^{\mu}=£_{\chi} \beta^{\mu}$ and $\delta_{\mathcal{X}} \Lambda^{\beta}=$ $\mathfrak{£}_{\chi} \Lambda^{\beta}-\mathfrak{f}_{\beta} \Lambda^{\chi}$. It may be explicitly checked that $\delta_{\mathcal{X}} \mu=$ $£_{\chi} \mu$ and therefore that $\mu$ is gauge invariant. Motivated by this, in the symmetry-unbroken phase of string fluids we consider the fields $\mathcal{B}=\left(\beta^{\mu}, \Lambda_{\mu}^{\beta}\right)$ and introduce the oneform chemical potential $\mu_{\mu}$ via the relation

$$
\frac{\mu_{\mu}}{T}=\Lambda_{\mu}^{\beta}+\beta^{\nu} b_{\nu \mu} .
$$

Given the transformation property $\delta_{\mathcal{X}} \Lambda_{\mu}^{\beta}=\mathfrak{£}_{\chi} \Lambda_{\mu}^{\beta}-\mathfrak{f}_{\beta} \Lambda_{\mu}^{\chi}$ under the action of $\mathcal{X}=\left(\chi^{\mu}, \Lambda_{\mu}^{\chi}\right)$, it is straightforward to check that $\delta_{\mathcal{X}} \mu_{\mu}=\mathfrak{£}_{\chi} \mu_{\mu}-T \partial_{\mu}\left(\beta^{\nu} \Lambda_{\nu}^{\chi}\right)$. Hence, unlike usual charged fluids, $\mu_{\mu}$ is not gauge invariant and cannot correspond to $\left(\varpi, h^{\mu}\right)$ of string fluids. Specifically, we cannot construct a gauge-invariant vector that would replace $h^{\mu}$ in Eq. (5) using just $\mu_{\mu}$.

In order to construct a gauge-invariant vector, we need to introduce a scalar field $\varphi$ that transforms in the nontrivial manner $\delta_{\mathcal{X}} \varphi=\chi^{\mu} \partial_{\mu} \varphi-\beta^{\mu} \Lambda_{\mu}^{\chi}$. This allows for the definition of the gauge-invariant combination given in Eq. (4). The scalar $\varphi$ is accompanied by its own equation of motion, which, following Ref. [15], reads $\delta_{\mathcal{B}} \varphi=\mathcal{O}(\partial)$ implying that $u^{\mu} h_{\mu}=\mathcal{O}(\partial)$. Using a part of the redefinition freedom in $\mu_{\mu}$, one may set $u^{\mu} h_{\mu}=0$ exactly, thus reproducing the variables of string fluids. We learn that instead of treating $\varpi$ and $h^{\mu}$ as fundamental hydrodynamic variables in string fluids, we should instead work with $\mu_{\mu}$ and $\varphi$. This also leads to well-defined equilibrium configurations given by $u^{\mu} / T=\delta_{t}^{\mu}$ and $\mu_{\mu} / T=b_{t \mu}$ along with $\varphi=\varphi_{0}$ which solves the expected ideal order Poisson's equation $\nabla_{\mu}\left(T \rho h^{\mu}\right)=0$, i.e., the no-monopole constraint of [6]

$$
\frac{1}{\sqrt{-g}} \partial_{\mu}\left(\sqrt{-g} \frac{\rho T^{2}}{\varpi} g^{\mu \nu}\left(b_{t \nu}-\partial_{\nu} \varphi_{0}\right)\right)=\mathcal{O}\left(\partial^{2}\right) .
$$

On this solution, the term in Eq. (6) vanishes. The introduction of $\varphi$ through Eq. (4) provides an improved version of the string fluids formulated in Ref. [3].

The transformation of $\varphi$ under the action of $\mathcal{X}$ involves the hydrodynamic field $\beta^{\mu}$, suggesting that, unlike usual superfluids, $\varphi$ is not a fundamental variable. In fact, in equilibrium, under a gauge transformation the scalar field transforms as $\varphi \rightarrow \varphi-\Lambda_{t}^{\chi}$, hinting that $\varphi$ might be better understood as the time component of a vector Goldstone mode $\varphi_{\mu}$, embedded into a larger theory in which the oneform symmetry is spontaneously broken.

One-form superfluids. - As pointed out in Refs. [8,10,11], the Goldstone mode corresponding to the spontaneous breaking of a one-form symmetry is a dynamical U(1) gauge field $\varphi_{\mu}$. Under the action of the set of one-form symmetry parameters $\mathcal{X}$, the Goldstone $\varphi_{\mu}$ transforms analogous to its zero-form counterpart

$$
\delta_{\mathcal{X}} \varphi_{\mu}=£_{\chi} \varphi_{\mu}-\Lambda_{\mu}^{\chi} .
$$

Thus, the scalar $\varphi$ appearing in Eq. (4) is in fact given by $\varphi=\beta^{\mu} \varphi_{\mu}$. We can define the gauge-invariant covariant derivative of $\varphi_{\mu}$,

$$
\xi_{\mu \nu}=2 \partial_{[\mu} \varphi_{\nu]}+b_{\mu \nu}
$$

which is a higher-form analogue of the superfluid velocity and transforms simply as $\delta_{\mathcal{X}} \xi_{\mu \nu}=£_{\chi} \xi_{\mu \nu}$.

The dynamics of one-form superfluids is also governed by Eq. (2) with constitutive relations written in terms of the hydrodynamic fields $T, u^{\mu}, \xi_{\mu \nu}$, and $\varpi h_{\mu}$ defined in Eq. (4), supplemented with the equation of motion for $\varphi_{\mu}$. Formulating the off-shell second law of thermodynamics analogous to zero-form superfluids [15], one can straightforwardly derive this equation at ideal order

$$
u^{\mu} \xi_{\mu \nu}=\varpi h_{\nu}+\mathcal{O}(\partial)
$$

This is a higher-form analogue of the Josephson equation for superfluids. Note that the condition $u^{\mu} h_{\mu}=\mathcal{O}(\partial)$ of string fluids follows from here. Using Eq. (11), we can remove $\varpi h_{\mu}$ from the independent set of hydrodynamic variables in favor of $\zeta_{\mu}=\xi_{\mu \nu} u^{\nu}$. Hence, the dynamics of one-form superfluids is governed by Eq. (2) alone, along with the off-shell second law of thermodynamics

$\nabla_{\mu} N^{\mu}=\frac{1}{2} T^{\mu \nu} \delta_{\mathcal{B}} g_{\mu \nu}+\frac{1}{2} J^{\mu \nu} \delta_{\mathcal{B}} \xi_{\mu \nu}+\Delta, \quad \Delta \geq 0$.

Here, $N^{\mu}=S^{\mu}+(1 / T) T^{\mu \nu} u_{\nu}-(1 / T) J^{\mu \nu} \zeta_{\nu}$ is the free energy current and 
$\delta_{\mathcal{B}} g_{\mu \nu}=2 \nabla_{(\mu} \beta_{\nu)}, \delta_{\mathcal{B}} \xi_{\mu \nu}=-2 \nabla_{[\mu}\left(\zeta_{\nu]} / T\right)+\beta^{\rho} H_{\rho \mu \nu}$

Equation (12) requires that for a given set of constitutive relations $\left(T^{\mu \nu}, J^{\mu \nu}\right)$ in terms of $\left(T, u^{\mu}, \xi_{\mu \nu}, g_{\mu \nu}\right)$, there must exist a free energy current $N^{\mu}$ and a positive semidefinite quadratic form $\Delta$ such that Eq. (12) is satisfied.

In order to discuss the constitutive relations of a one-form superfluid in four spacetime dimensions, we decompose $\xi_{\mu \nu}=2 u_{[\mu} \zeta_{\nu]}-\epsilon_{\mu \nu \rho \sigma} u^{\rho} \bar{\zeta}^{\sigma}$. A generic one-form superfluid can depend on both $\zeta_{\mu}$ and $\bar{\zeta}_{\mu}$ arbitrarily but here we mention two special cases that find a direct application in plasma physics. The "string fluid limit" is the case in which the constitutive relations depend on $\zeta_{\mu}=-\varpi h_{\mu}$ but not on $\bar{\zeta}_{\mu}$, which, as we show below, is dual to MHD. Formally, removing $\bar{\zeta}_{\mu}$ from the constitutive relations means that the one-form symmetry is only broken along the timelike direction $\beta^{\mu}$ while the spatial part of the symmetry is left intact. This gives rise to the improved string fluid theory described earlier where $\varphi=\beta^{\mu} \varphi_{\mu}$ is introduced according to Eq. (4). Another interesting case is the "electric limit," in which the hierarchy of gradients $\zeta_{\mu}=\mathcal{O}(1)$ and $\bar{\zeta}_{\mu}=\mathcal{O}(\partial)$ is assumed. This latter case, where the full one-form symmetry is broken, can be shown to be equivalent to plasma in the absence of free charges [12]. Focusing on the former string fluid limit, we note that there are two Lorentz and gauge invariant scalars at ideal order, namely, $T$ and $\varpi=\sqrt{\zeta_{\mu} \zeta^{\mu}}$, on which the free energy current $N^{\mu}=N(T, \varpi) \beta^{\mu}$ can depend. Using Eq. (12) we find the ideal order constitutive relations Eq. (5). Thus, at ideal order, one-form superfluids in this limit reduce to string fluid dynamics, which continues to be the case at higher orders.

One-derivative string fluids. - We parametrize the nonhydrostatic corrections to the one-form superfluid constitutive relations as

$$
\begin{aligned}
\delta T_{(1)}^{\mu \nu} & =\delta f \Delta^{\mu \nu}+\delta \tau h^{\mu} h^{\nu}+2 \ell^{(\mu} h^{\nu)}+t^{\mu \nu}, \\
\delta J_{(1)}^{\mu \nu} & =2 m^{[\mu} h^{\nu]}+s^{\mu \nu} .
\end{aligned}
$$

All the tensor structures appearing here are transverse to $u^{\mu}$ and $h^{\mu}$. Recall that we had used part of the redefinition freedom in $\mu_{\mu}$ around Eq. (4) to set $u^{\mu} h_{\mu}=0$. In writing Eq. (14), we also used the residual freedom in $\mu_{\mu}$ along with that in $u^{\mu}$ and $T$ to work in an analogue of the "Landau frame" and set $u_{\mu} \delta T_{(1)}^{\mu \nu}=u_{\mu} \delta J_{(1)}^{\mu \nu}=0$. When working with full one-form superfluids, we can instead choose to use the redefinition of $\mu_{\mu}$ to make Eq. (11) exact, at the expense of having $u_{\mu} \delta J_{(1)}^{\mu \nu} \neq 0[12]$.

Restring ourselves to derivative corrections that respect $C P$ invariance [16], in the nonhydrostatic sector we find

$$
\begin{aligned}
\delta f & =-T / 2\left(\zeta_{\perp} \Delta^{\mu \nu}+\zeta_{\times} h^{\mu} h^{\nu}\right) \delta_{\mathcal{B}} g_{\mu \nu}, \\
\delta \tau & =-T / 2\left(\zeta_{\times}^{\prime} \Delta^{\mu \nu}+\zeta_{\|} h^{\mu} h^{\nu}\right) \delta_{\mathcal{B}} g_{\mu \nu}, \\
\ell^{\mu} & =-T\left(\eta_{\|} \Delta^{\mu \sigma}+\tilde{\eta}_{\|} \epsilon^{\mu \sigma}\right) h^{\nu} \delta_{\mathcal{B}} g_{\sigma \nu}, \\
m^{\mu} & =-T\left(r_{\perp} \Delta^{\mu \sigma}+\tilde{r}_{\perp} \epsilon^{\mu \sigma}\right) h^{\nu} \delta_{\mathcal{B}} \xi_{\sigma \nu} \\
t^{\mu \nu} & =-T\left(\eta_{\perp} \Delta^{\rho \mu \mu} \delta_{\mathcal{B}} g_{\rho \sigma}-\tilde{\eta}_{\perp} \epsilon^{\rho\langle\mu}\right) \Delta^{\nu\rangle \sigma} \delta_{\mathcal{B}} g_{\rho \sigma}, \\
s^{\mu \nu} & =-\operatorname{Tr}_{\|} \Delta^{\mu \lambda} \Delta^{\nu \sigma} \delta_{\mathcal{B}} \xi_{\lambda \sigma},
\end{aligned}
$$

where $\epsilon^{\mu \nu}=\epsilon^{\mu \nu \rho \sigma} u_{\rho} h_{\sigma}$. Using Eqs. (12), we obtain exactly the same constraints and number of dissipative transport coefficients as for string fluids I in Refs. [3,4].

The hydrostatic sector of the theory has not been considered in Refs. [3,4]. This sector is described by a hydrostatic effective action for the Goldstone mode. Aligning the fluid velocity with a timelike Killing vector, up to first order in derivatives, this action is

$\mathcal{S}=\int d^{4} x \sqrt{-g}\left(p-\frac{\alpha}{6} \epsilon^{\mu \nu \lambda \sigma} u_{\mu} H_{\nu \lambda \sigma}-\beta \epsilon^{\mu \nu} \partial_{\mu} u_{\nu}\right)$,

where $\alpha(T, \varpi)$ and $\beta(T, \varpi)$ are hydrostatic transport coefficients. Contrary to Refs. [3,5], no assumptions regarding the presence of spatial isometries in the background are necessary. Extremizing Eq. (16) with respect to $\varphi$ yields its equation of motion in equilibrium, which improves Eq. (8) due to the $\alpha$ and $\beta$. Equation (16) characterizes all equilibrium configurations of MHD and guarantees that all nonhydrostatic contributions in Eq. (15) vanish. We will now show that one-form superfluidity in the string fluid limit is exactly equivalent to MHD.

MHD/string fluid correspondence.-The dynamics of MHD is determined by the equations of motion (1) where $A_{\mu}$ is a dynamical gauge field. In this setting, $B^{\mu}$ is treated as $\mathcal{O}(1)$ while $E^{\mu}$ as $\mathcal{O}(\partial)$. The constitutive relations of MHD are solutions of the off-shell second law of thermodynamics

$\nabla_{\mu} N_{\mathrm{MHD}}^{\mu}=\frac{1}{2} T^{\mu \nu} \delta_{\mathcal{B}} g_{\mu \nu}+J^{\mu} \delta_{\mathcal{B}} A_{\mu}+\Delta, \quad \Delta \geq 0$.

Recalling that $J^{\mu \nu}=\frac{1}{2} \epsilon^{\mu \nu \rho \sigma} F_{\rho \sigma}$ and $J_{\mathrm{ext}}^{\mu}=\frac{1}{6} \epsilon^{\mu \nu \rho \sigma} H_{\nu \rho \sigma}$ together with

$$
N^{\mu}=N_{\mathrm{MHD}}^{\mu}-\frac{1}{T} J^{\mu \nu} \zeta_{\nu}+\frac{\mu}{T} J_{\mathrm{ext}}^{\mu},
$$

it follows that Eq. (17) in MHD is equivalent to Eq. (12), provided that Maxwell's equations, $J^{\mu}+J_{\text {ext }}^{\mu}=0$, are taken on shell. Formally, this requires solving for $\mu$ and $E^{\mu}$ using Maxwell's equations, after which the remaining fields $u^{\mu}, T$, and $B^{\mu}$ can be mapped to $u^{\mu}, T, \varpi$, and $h^{\mu}$ of string fluids, modulo hydrodynamic frame transformations [12]. Given that identification of Eqs. (17) and (12), the 
equivalence between the most generic constitutive relations allowed by it also follows.

As an example, we consider the first order charge current $J^{\mu}$ in parity-invariant MHD [4]

$$
\begin{aligned}
J^{\mu}= & \left(q+\frac{\partial M_{\Omega}}{\partial \mu} \epsilon^{\lambda \nu \rho \sigma} B_{\lambda} u_{\nu} \partial_{\rho} u_{\sigma}\right) u^{\mu}-\epsilon^{\mu \nu \rho \sigma} \partial_{\rho}\left(\varpi u_{\nu} \hat{B}_{\sigma}\right) \\
& +\left(\sigma_{\perp} \mathbb{B}^{\mu \nu}+\sigma_{\|} \hat{B}^{\mu} \hat{B}^{\nu}+\tilde{\sigma} \epsilon^{\mu \nu \rho \sigma} u_{\rho} \hat{B}_{\sigma}\right) V_{\nu}+\mathcal{O}\left(\partial^{2}\right),
\end{aligned}
$$

where $M_{\Omega}$ is a hydrostatic transport coefficient while $\sigma_{\perp}$, $\sigma_{\|}$, and $\tilde{\sigma}$ are dissipative ones. The remaining parameters obey the thermodynamic relations $d P=s d T+q d \mu-$ $\varpi d|B|$, where $P$ is the fluid pressure in MHD. We have also defined $\hat{B}^{\mu}=B^{\mu} /|B|, \mathbb{B}^{\mu \nu}=g^{\mu \nu}+u^{\mu} u^{\nu}-\hat{B}^{\mu} \hat{B}^{\nu}$, and $V^{\mu}=E^{\mu}-T P^{\mu \nu} \partial_{\nu}(\mu / T)$. Projecting Eq. (19) along $u^{\mu}$, we have that $q\left(T, \mu, B^{2}\right)=\mathcal{O}(\partial)$, which can be formally solved by $\mu=\mu_{0}\left(T, B^{2}\right)+\mathcal{O}(\partial)$ [17]. In turn, one can determine $E^{\mu}$ by projecting Eq. (19) transverse to $u^{\mu}$. Comparing this result with $F^{\mu \nu}=-\frac{1}{2} \epsilon^{\mu \nu \rho \sigma} J_{\rho \sigma}$ together with Eq. (15), we can identify $B^{\mu}=\rho h^{\mu}+\mathcal{O}(\partial)$ and the transport coefficients

$$
\begin{gathered}
\alpha=\mu_{0}, \quad \beta=\rho M_{\Omega}+\varpi \alpha, \quad r_{\perp}=\frac{\sigma_{\perp}}{\sigma_{\perp}^{2}+\tilde{\sigma}^{2}}\left(\frac{s T}{\epsilon+p}\right)^{2}, \\
\tilde{r}_{\perp}=\left(\frac{s T}{\epsilon+p}\right)^{2}\left(\frac{-\tilde{\sigma}}{\sigma_{\perp}^{2}+\tilde{\sigma}^{2}}+\frac{2 \rho \alpha}{s T}\right), \quad r_{\|}=\frac{1}{\sigma_{\|}},
\end{gathered}
$$

where $p=P+\varpi \rho$. The remaining transport coefficients in Eq. (15) can be identified by comparing the stress tensor $T^{\mu \nu}$ in the two formulations. Using the results of Ref. [4], explicitly, we find

$$
\begin{array}{ll}
\zeta_{\perp}=\zeta_{1}-\frac{2}{3} \eta_{1}, & \zeta_{\times}=\zeta_{1}+\zeta_{2}-\frac{2}{3} \eta_{1}-\frac{2}{3} \eta_{2}, \\
\zeta_{\times}^{\prime}=\zeta_{1}+\frac{4}{3} \eta_{1}, & \zeta_{\|}=\zeta_{1}+\zeta_{2}+\frac{4}{3} \eta_{1}+\frac{4}{3} \eta_{2},
\end{array}
$$

where $\zeta_{1}, \zeta_{2}, \eta_{1}$, and $\eta_{2}$ have been defined in Ref. [4]. The other four coefficients $\eta_{\perp}, \eta_{\|}, \tilde{\eta}_{\perp}$, and $\tilde{\eta}_{\|}$map one-toone with those denoted by the same symbols in Ref. [4]. Hence, we see that at one-derivative order, MHD is entirely equivalent to one-form superfluids in the string fluid limit.

Note that the equivalence between the two formulations, even just in the dissipative sector, required the presence of the hydrostatic coefficient $\alpha$ in one-form superfluids, which captures a nonzero $\mu=\mu_{0}$ in an MHD configuration. This coefficient was absent in all the previous discussions on string fluids [2-4], thereby the equivalence only holding in a state with $\mu_{0}=0$ of MHD. Furthermore, the authors of Ref. [4] focused only on the dissipative sector and considered this equivalence at the linearized level [18]. However, here we have provided the exact nonlinear map between transport coefficients, including the hydrostatic sector which had not been previously analyzed.

Outlook.-In this Letter we have formulated a theory of one-form superfluidity and illustrated how MHD can be understood as a particular sector of this theory. This dual formulation is in many ways a better and cleaner description of MHD as it makes all the global symmetries of MHD manifest, eliminates the nonpropagating fields $\mu$ and $E^{\mu}$, and the electric fields being $\mathcal{O}(\partial)$ becomes a consequence rather than an assumption. Most importantly, unlike the conventional formulation, in the superfluid formulation, the constitutive relations are directly obtained for the physically observable electromagnetic fields, which considerably simplifies the computation of correlation functions.

This description of MHD as superfluidity is entirely based on conservation equations, even when including dissipation effects. Together with the understanding of the necessary conditions for equilibrium, it can provide initial configurations for obtaining interesting insights in the context of astrophysical phenomena using numerical simulations (e.g., Ref. [19]). As a proof of concept, we obtain an equilibrium configuration (i.e., without dissipation) of a slowly rotating magnetized star on a flat background $g_{\mu \nu}=\eta_{\mu \nu}, b_{\mu \nu}=0$. The equilibrium configuration corresponds to $u^{\mu} / T=\delta_{t}^{\mu}+\omega\left(y \delta_{x}^{\mu}-x \delta_{y}^{\mu}\right)$ and $\mu_{\mu} / T=0$, where $\omega$ is a small angular velocity along the $z$ axis. Assuming an equation of state $p=p_{m}(T)-(1 / 2 \chi) \varpi^{2}$ with constant magnetic susceptibility $\chi$, we can find a solution of Eq. (8) for the scalar Goldstone as $\varphi_{0}=-z \cos \alpha+(x-\omega t y)$ $\sin \alpha+\mathcal{O}\left(\omega^{2}\right)$, leading to $\varpi h_{\mu}=\cos \alpha \delta_{\mu}^{z}-\sin \alpha\left(\delta_{\mu}^{x}-\right.$ $\left.\omega y \delta_{\mu}^{t}-\omega t \delta_{\mu}^{y}\right)+\mathcal{O}\left(\omega^{2}\right)$. The angle $\alpha$ parametrizes the misalignment of the magnetic axis (direction of strings) with the rotational axis of the star. For a nonzero $\alpha$, the vector $h_{\mu}$ is not aligned along a spacelike isometry neither with a linear combination of isometries as in Refs. [3,9], and hence necessitates the new theory that we have presented in this Letter. We also note that in the traditional formulation of MHD, one would be required to solve Maxwell's equations for equilibrium configurations of $A_{\mu}$, and typically simplifications such as working within the force-free electrodynamics (FFE) regime are imposed. On the other hand, using the framework proposed here, we have reduced the problem of finding equilibrium configurations to the problem of finding solutions to a scalar Poisson's equation (8). This is a considerable leap forward towards providing appropriate initial conditions for numerical simulations in arbitrary spacetime backgrounds. More generally, we expect this theory to be useful for obtaining new analytic equilibrium solutions for accretion disks surrounding Kerr black holes or magnetised stars such as pulsars, and to probe mechanisms of energy transport therein by studying fluctuations around such solutions including the effects of dissipation. 
We would like to thank J. Bhattacharya, J. Hernandez, and specially N. Iqbal for various helpful discussions. We would also like to thank J. Bhattacharya, S. Grozdanov, and N. Iqbal for comments on an earlier draft. J. A. is partly supported by the Netherlands Organization for Scientific Research (NWO). A. J. would like to thank the Perimeter Institute, where part of this project was done, for hospitality. A. J. is supported by the Durham Doctoral Scholarship offered by Durham University.

*j.armas@uva.nl

†akash.jain@durham.ac.uk

[1] J. Goedbloed and S. Poedts, Principles of Magnetohydrodynamics: With Applications to Laboratory and Astrophysical Plasmas (Cambridge University Press, Cambridge, England, 2004).

[2] D. Schubring, Phys. Rev. D 91, 043518 (2015).

[3] S. Grozdanov, D. M. Hofman, and N. Iqbal, Phys. Rev. D 95, 096003 (2017).

[4] J. Hernandez and P. Kovtun, J. High Energy Phys. 05 (2017) 001.

[5] J. Armas, J. Gath, A. Jain, and A. V. Pedersen, J. High Energy Phys. 05 (2018) 192.
[6] C. F. Gammie, J. C. McKinney, and G. Toth, Astrophys. J. 589, 444 (2003).

[7] J. A. Font, Living Rev. Relativity 11, 7 (2008).

[8] D. Gaiotto, A. Kapustin, N. Seiberg, and B. Willett, J. High Energy Phys. 02 (2015) 172.

[9] M. M. Caldarelli, R. Emparan, and B. Van Pol, J. High Energy Phys. 04 (2011) 013.

[10] E. Lake, arXiv:1802.07747.

[11] D. M. Hofman and N. Iqbal, SciPost Phys. 6, 006 (2019).

[12] J. Armas and A. Jain, arXiv:1811.04913.

[13] S. Bhattacharyya, S. Jain, S. Minwalla, and T. Sharma, J. High Energy Phys. 01 (2013) 040.

[14] F. M. Haehl, R. Loganayagam, and M. Rangamani, J. High Energy Phys. 05 (2015) 060.

[15] A. Jain, Phys. Rev. D 95, 121701(R) (2017).

[16] Parity $P$ acts on various quantities as usual, while charge conjugation $C$ flips the sign of $J^{\mu \nu}, b_{\mu \nu}$, and $h^{\mu}$.

[17] In making these identifications, we have assumed $J_{\text {ext }}^{\mu}$ to be $\mathcal{O}(\partial)$, which means that $J^{\mu}=\mathcal{O}(\partial)$ on shell.

[18] The authors of Ref. [4] also assumed $-2 B^{2} \partial P / \partial B^{2} \ll$ $\epsilon+P$, which implies that $\epsilon+p \approx s T$. In this regime, Eq. (20) reduces to $r_{\perp}=\sigma_{\perp} /\left(\sigma_{\perp}^{2}+\tilde{\sigma}^{2}\right)$ and $\tilde{r}_{\perp}=-\tilde{\sigma}_{\perp}^{2} /$ $\left(\sigma_{\perp}^{2}+\tilde{\sigma}^{2}\right)$, as found in Ref. [4].

[19] R. F. Penna, J. C. McKinney, R. Narayan, A. Tchekhovskoy, R. Shafee, and J. E. McClintock, Mon. Not. R. Astron. Soc. 408, 752 (2010). 